\title{
Scalable soft real-time supervisor for tomographic AO
}

N. Doucet, R. Kriemann, E. Gendron, D. Gratadour, H. Ltaief, et al.

N. Doucet, R. Kriemann, E. Gendron, D. Gratadour, H. Ltaief, D. Keyes, "Scalable soft real-time supervisor for tomographic AO," Proc. SPIE 10703, Adaptive Optics Systems VI, 107034L (17 July 2018); doi: $10.1117 / 12.2313273$

SPIE Event: SPIE Astronomical Telescopes + Instrumentation, 2018, Austin, Texas, United States 


\title{
Scalable Soft Real-Time Supervisor for Tomographic AO
}

\author{
Doucet N. ${ }^{\mathrm{a}, \mathrm{b}}$, Kriemann R. ${ }^{\mathrm{c}}$, Gendron E. ${ }^{\mathrm{a}}$, Gratadour D. ${ }^{\mathrm{a}}$, Ltaief H. ${ }^{\mathrm{b}}$, and Keyes D. ${ }^{\mathrm{b}}$ \\ ${ }^{a}$ LESIA - Observatoire de Paris/ Université Paris Diderot \\ ${ }^{\mathrm{b}}$ Extreme Computing Research Center - KAUST \\ ${ }^{\mathrm{c}}$ Max Planck Institute for Math. i.t.S. - Leipzig
}

\begin{abstract}
Implementations of AO tomography for the next generation of Extremely Large Telescopes (ELTs) is challenging because of the extremely large number of degrees of freedom of such systems, in particular when it comes to the tomographic reconstructor computation, due to its size. The computation of this matrix, via the supervisor module, requires leveraging high performance computing techniques, on shared or distributed memory systems, to comply with the specifications of tomographic AO systems, which prescribe an update rate of the order of few minutes.

In the scope of the Green-Flash project, we are exploring several approaches to optimize the execution of this soft real-time supervision pipeline. This includes low-rank techniques to reduce the computational load. We have tested several compression schemes to optimize the linear algebra involved in the tomographic reconstructor as well as the computation of the covariance matrices involved in this process.

We present, in this paper, the scalable and portable pipeline we have developed to address these issues. Performance in terms of time to solution and scalability are reported. Additionally, the case of low-rank algorithms is stressed as both an attempt to address the computation challenge of the tomographic reconstructor for the supervisor module, and a way to reduce the computational load (hence the overall RTC system latency) at the level of the real-time data pipeline.
\end{abstract}

\section{INTRODUCTION}

As part of the Green-Flash project, we design a loop supervision module for tomographic Adaptive Optic (AO) systems at the E-ELT scale. We aim in particular to provide such systems with tomographic reconstructors, using the Learn and Apply method ${ }^{1}$. At the ELT scale, one has to deal with very large matrices sizes (of the order of $100 k)$. Furthermore, the reconstructor needs to be updated frequently, at the minute rate. In order to cope with these constraints, we propose to apply Hierarchical matrices and low-rank techniques to the Apply step of the pipeline for computing the tomographic reconstructor, as well as the matrix generation. We first derive a general approach to compute the tomographic reconstructor (ToR) for several AO concepts, including Ground Layer (GL)AO, Laser Tomography (LT)AO, Multi-Conjugate (MC)AO and Multi-Object (MO)AO. While we show that matrix generation time dominates the execution profile of the pipeline for several of these AO modes, we report on the preliminary results obtained so far with low-rank techniques.

\section{AO MODES}

The software we are currently developing aims at providing an optimized loop supervision strategy for several $\mathrm{AO}$ flavors. This work is based on the formalism detailed in a previous paper describing a pseudo analytical approach $\left[^{2}\right]$ for $\mathrm{AO}$ simulations and generalized to several tomographic $\mathrm{AO}$ configurations. In each AO case, detailed below, multiple wave front sensors (WFS) are used. The difference lies in the number of truth sensors (TS), targets and deformable mirrors (DM) in the system. We consider WFS the actual sensors, TS the fictive, and so-called truth, sensors toward a direction of interest, and targets the objects to be observed. Measurements

Further author information: (Send correspondence to N. Doucet)

N. doucet: E-mail: nicolas.doucet@observatoiredeparis.psl.eu, Telephone: +33(0)145077786

This work is sponsored through a grant from project \#671662, a.k.a. Green Flash, funded by European Commission under program H2020-EU.1.2.2 coordinated in H2020-FETHPC-2014

Adaptive Optics Systems VI, edited by Laird M. Close, Laura Schreiber, Dirk Schmidt, Proc. of SPIE Vol. 10703, 107034L · @ 2018 SPIE CCC code: $0277-786 \mathrm{X} / 18 / \$ 18 \cdot$ doi: $10.1117 / 12.2313273$

Proc. of SPIE Vol. 10703 107034L-1 
from the WFS are labeled $m$, those from the TS are labeled $t_{i}$ or simply $t$, if only one TS is used. Covariance matrices between measurements are computed for every approach: the covariance matrix between the WFS measurements is notated to as $\langle m, m\rangle$ and the covariance matrix between the WFS and the TS will be written $<t_{i}, m>$.

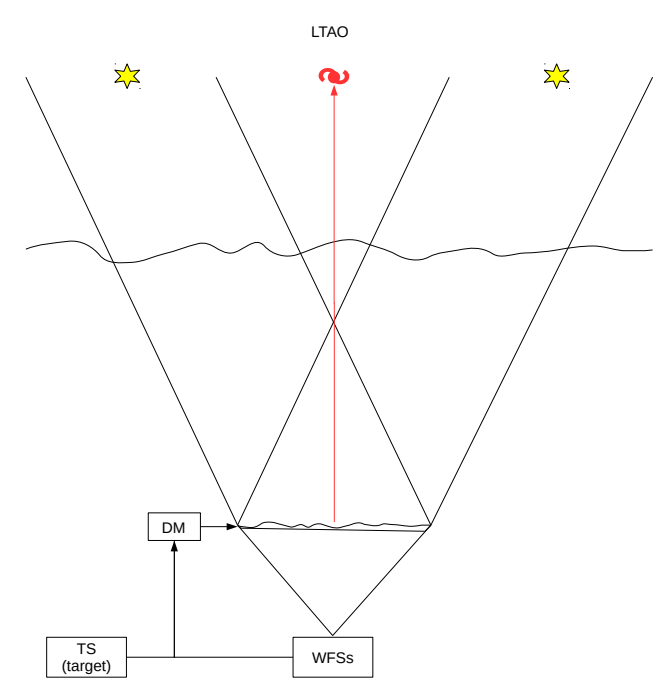

(a)

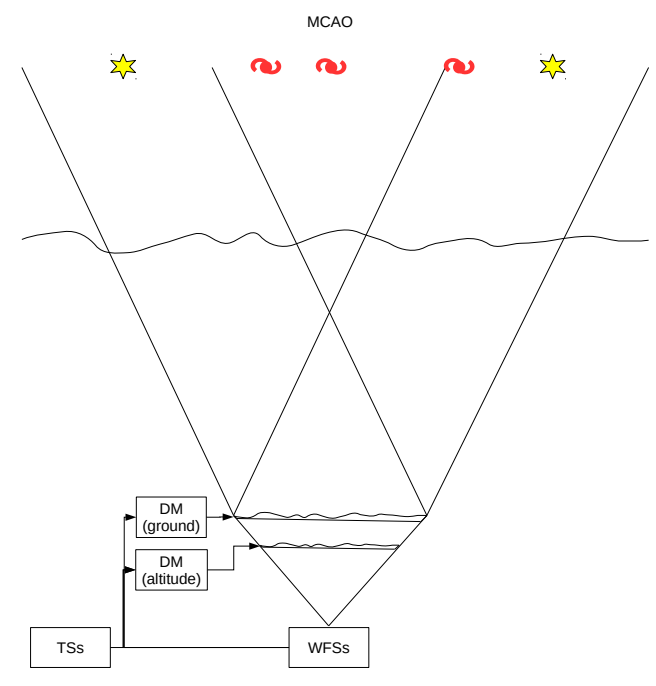

(c)

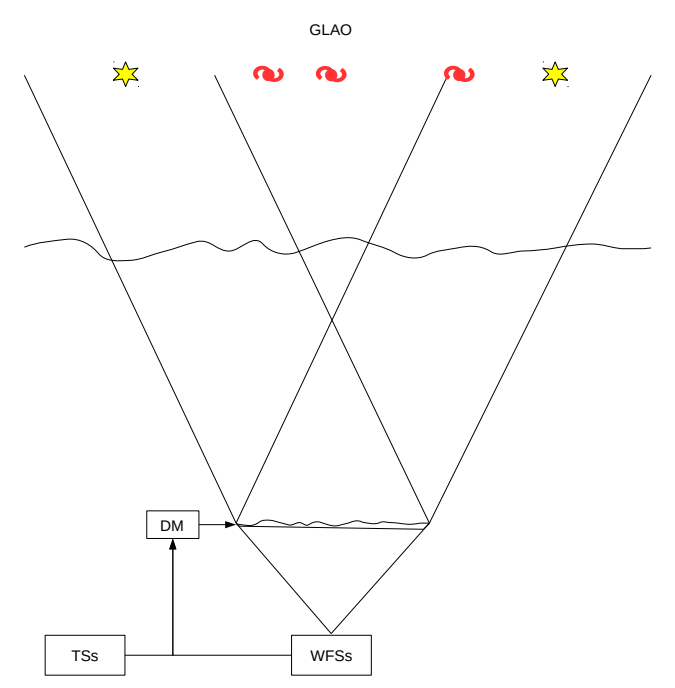

(b)

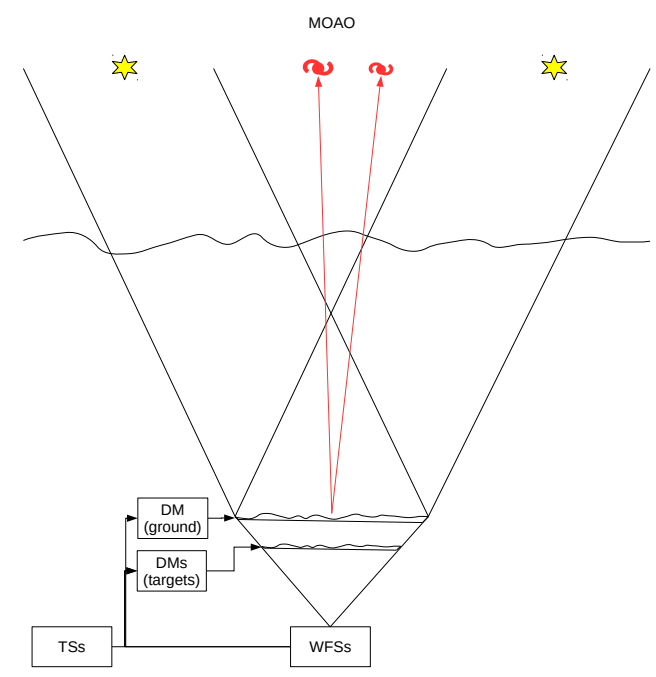

(d)

Figure 1: (a) LTAO: correct the turbulences in the observation direction with a single DM. (b) GLAO: correct the whole field of view with a single DM using multiple TS. (c) MCAO: correct the whole field of view with two DMs using multiple TS. (d) MOAO: correct the turbulent in multiple directions.

\subsection{Tomographic Reconstructor}

All the tomographic AO approaches described below rely on the use of a tomographic reconstructor $\left[^{2}\right]$ to provide, from the WFS measurement, the command vector to be applied on the DM. This reconstructor, which can be 
considered as a change of basis matrix, is computed as follows:

$$
M=D^{\dagger} \times R,
$$

with:

$$
R=<t, m>\times<m, m>^{-1},
$$

and where $D^{\dagger}$ is a change of basis matrix from the TS measurements to the DM's actuators. This matrix is typically computed as the pseudo-inverse of the interaction matrix $D$ between the TS and the DM's actuators, and obtained as follows:

$$
D^{\dagger}=\left(D^{t} D\right)^{-1} D^{t}
$$

\subsection{LTAO}

The LTAO mode aims at correcting the turbulence in the particular direction of a single target, using a single DM and from the measurements of a fixed number of sensors. One TS is considered in the direction of the target of interest.

After computing the covariance matrices $\langle m, m\rangle$ and $\langle t, m\rangle$, the reconstructor $M$ is computed with respect to Equations (1) and (2). This is the simplest mode presented in this document.

\subsection{GLAO}

The GLAO mode aims at compensating for the effect of the turbulent layer at the ground level, using a single DM for targets distributed over the whole field of view. In order to take into account these multiple targets, we use a grid of TS distributed regularly over the whole field of view and we average the measurements from theses TSs. We propose to compute the reconstructor as follows:

$$
M=\mathscr{D}^{\dagger} \times<T, m>\times<m, m>^{-1} .
$$

Despite the similarity with the formulation (2), the matrices $\mathscr{D}^{\dagger}$ and $\langle T, m\rangle$ are in fact "meta-matrices" which are the concatenation of the matrices $\left(D^{\dagger}\right.$ and $<t_{i}, m>$ ) for all the TSs:

$$
\begin{gathered}
\mathscr{D}^{\dagger}=\left[\begin{array}{lll}
D^{\dagger} & \ldots & D^{\dagger}
\end{array}\right], \\
<T, m>=\left[\begin{array}{c}
<t_{1}, m> \\
\vdots \\
<t_{N}, m>
\end{array}\right] .
\end{gathered}
$$

We show in the following that solving Equation (4) is equivalent to solve Equation (2) using the average of the TS measurements. The DM being conjugated at the ground layer, the interaction matrices of each TS are identical and therefore the meta-interaction matrix can be defined as:

$$
\mathscr{D}=\left[\begin{array}{c}
D \\
\vdots \\
D
\end{array}\right]
$$

then $\mathscr{D}^{\dagger}$ is computed with Equation (3)

$$
\begin{gathered}
\left(\mathscr{D} \mathscr{D}^{t}\right)=\left[\begin{array}{lll}
D^{t} & \ldots & D^{t}
\end{array}\right]\left[\begin{array}{c}
D \\
\vdots \\
D
\end{array}\right]=N\left(D^{t} D\right) \\
\left(\mathscr{D}^{t}\right)^{-1}=\frac{1}{N}\left(D^{t} D\right)^{-1}
\end{gathered}
$$




$$
\mathscr{D}^{\dagger}=\frac{1}{N}\left[\begin{array}{lll}
D^{\dagger} & \ldots & D^{\dagger}
\end{array}\right]
$$

finally, using this last result in Equation (4)

$$
\begin{gathered}
M=\frac{1}{N}\left[\begin{array}{lll}
D^{\dagger} & \ldots & D^{\dagger}
\end{array}\right]\left[\begin{array}{c}
<t_{1}, m> \\
\vdots \\
<t_{N}, m>
\end{array}\right]<m, m>^{-1}, \\
M=D^{\dagger}\left(\frac{1}{N} \sum_{i=1}^{N}<t_{i}, m>\right)<m, m>^{-1} .
\end{gathered}
$$

We obtain the average mentioned above in the last formulation of the reconstructor.

\subsection{MCAO}

In this approach, multiple DMs conjugated at different but fixed altitudes are used to compensate turbulence over the whole field of view concurrently. In the following, we show how our approach can be generalized for two $\mathrm{DMs}$ in the MCAO case, one of which being conjugated at the ground (hence with a similar behavior than the GLAO DM).

In this case, the tomographic reconstructor can be obtained by solving the Equation (4), rewriting the command matrix as a meta-command matrix.

Since the first DM is conjugated at the ground layer, the interaction matrices of this DM with all the TS are equal, noted $D 0$. Unfortunately, this property does not hold for the second DM and each TS has its own interaction matrix $D_{i}$. The overall interaction matrix is thus written as:

$$
\mathscr{D}=\left[\begin{array}{cc}
D & D_{1} \\
\vdots & \vdots \\
D & D_{N}
\end{array}\right]
$$

and the command matrix will be of the form:

$$
\mathscr{D}^{\dagger}=\left[\begin{array}{ccc}
D g_{1}^{\dagger} & \cdots & D g_{N}^{\dagger} \\
D a_{1}^{\dagger} & \cdots & D a_{N}^{\dagger}
\end{array}\right] .
$$

Here, we note that the $D g_{i}^{\dagger}$ are not the pseudo inverse of the $D$ nor $D a_{i}^{\dagger}$ the pseudo inverse of the $D_{i}$. This partitioning can only be performed after the pseudo-inversion process. However, by replacing in the Equation (4), the matrix can be expressed as:

$$
M=\sum_{i=1}^{N}\left[\begin{array}{l}
D g_{i}^{\dagger} \times<t_{i}, m> \\
D a_{i}^{\dagger} \times<t_{i}, m>
\end{array}\right]<m, m>^{-1} .
$$

This reconstructor matrix will provide the commands to be applied to both DMs from the WFS measurements.

\subsection{MOAO}

In the case of MOAO, multiple targets are associated to multiple DMs and the wide field correction is obtained only in small island of interest, defined by each target's location. An additional DM, most likely conjugated at the ground layer, is used to provide a first stage of compensation, for all the targets in the FoV (similarly to GLAO). The wide field reconstructor $M g$, used to compute the commands to be applied to the ground layer DM, is computed as a GLAO reconstructor (4). The second reconstructor, used to compute the commands to be applied to each individual DM in the target specific channels, has to compensate only for what is not done by the wide field correction, therefore as mentioned in $\left[^{3}\right]$ the Equation (1) is modified into:

$$
M_{i}=D_{i}^{\dagger}\left(R_{i}-D_{g} M_{g}\right) .
$$

With $D_{g}$ the interaction matrix of the wide field DM and $D_{i}^{\dagger}$ the command matrix of the DM associated to the target $i$. 


\subsection{Execution profile}

We profiled the execution of the different parts of the pipeline for the LTAO, GLAO and MCAO. The idea here is to identify the most time consuming component of the pipeline, so we set synchronization points between the different parts. The servers used for computation are referred to with their names, their specifications are detailed at the end of the document (Tab.7). The simulated telescope has a diameter of 39 meters and 10 WFS with a number of measurements of $90 k$, the LTAO uses a single TS in the observation direction whereas the GLAO and MCAO have a total of 24 TSs scattered in the whole field of view. The computation time of the

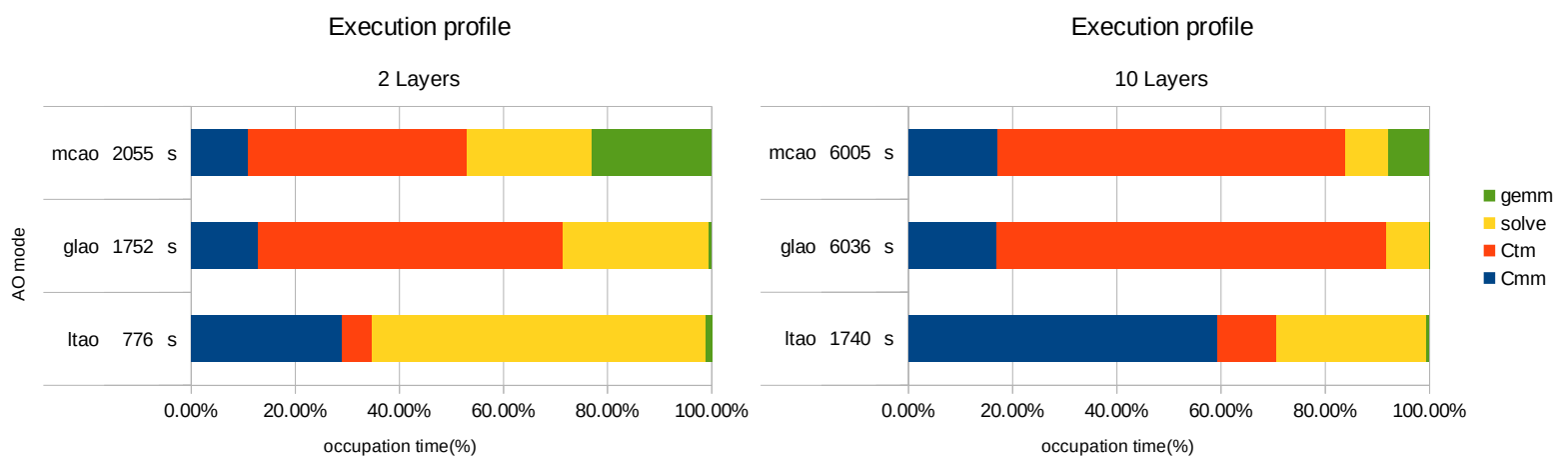

Figure 2: Pipeline execution profile for the LTAO, GLAO and MCAO (on Nirvana).

covariance matrices depends on the number of atmospheric layers taken into account, while the linear algebra parts depend only on the matrices size. As the number of layers increases, the matrix generation becomes the dominating part, up to $90 \%$. Therefore, in order to improve the time to solution of this pipeline, we need to improve this specific step.

\section{HIERARCHICAL TECHNIQUES FOR MATRIX GENERATION}

For each of the AO modes above, several covariance matrices have to be generated, and at ELT scale, it represents a large portion of the time compare to the resolution of Equation (1) when the number of matrices to generate grows $\mathrm{up}^{4}$. In order to speed up the application, we explored a low-rank approach and present the first results hereafter, in terms of compression rate (defined as memory(compressed)/memory(dense)), execution time and error to solution. These results were obtained with the library HLIBpro ${ }^{5}$.

In the following, we consider a LTAO system with a single target. For convenience, we note $C_{m m}$ the covariance matrix $<m, m>$ and $C_{t m}$ the matrix $<t, m>$.

\subsection{Hierarchical matrix format}

A hierarchical matrix $\left(\mathcal{H}\right.$-matrix $\left.{ }^{6,7}\right)$ is a storage format for compressible dense matrices, which also permits full matrix arithmetic. $\mathcal{H}$-matrices are based on an hierarchical partitioning of the index sets for the rows and columns of the dense matrix. These hierarchical partitionings together with an admissibility condition enable the identification of compressible matrix blocks. For those subblocks low-rank approximations are computed. For all non-compressible blocks of the matrix, the original dense representation is used.

The rank of the low-rank approximation is either predefined or adaptively chosen based on a given accuracy. Furthermore, low-rank blocks are represented in the factored form $M=A \cdot B^{T}$ with $M \in \mathbb{R}^{n \times m}, A \in \mathbb{R}^{n \times k}, B \in$ $\mathbb{R}^{m \times k}$ and $k$ being the rank of the low-rank matrix.

In addition to the $\mathcal{H}$-matrix format described above, we tested a block low-rank format ${ }^{8}$, and another format we will refer as "refin". The block low-rank format is a particular case of $\mathcal{H}$-matrices, in which the representation is flat in the way that there is no nested blocks, in addition, the clustering is regular hence all blocks are the result of the same subdivision. The "refine" format, was developed by Ronald Kriemann as an attempt to use 
the block rank after low-rank approximation to identify incompressible blocks and to further subdivide those blocks to permit low-rank compression on sub blocks.

The matrix generation is performed using Adaptive Cross Approximation Algorithm ${ }^{9}$. This algorithm iteratively generates a low-rank approximation of a matrix by computing single pairs of matrix rows/columns, thereby only evaluating a small amount of the matrix elements.

\subsection{Apply pipeline}

The last step of the Learn and Apply consists in solving Equation (2), written as:

$$
C_{m m} \times R=C_{t m} .
$$

We assessed the capabilities of the low-rank techniques for this task. However the results (Fig. 3) of the arithmetic is slower using compressed format compared to the dense operations. This behavior would suggest a rank growth during the arithmetic operations. The compression rate will confirm this assumption. It decreases after both the factorization and the direct solve, ending up worst than the dense approach. The rank growth on theses operations can be held responsible for such behavior. As a result, the Hierarchical formalism does not seem suitable to solve the system at hand.

The presented approach aims to use the most performant method for each component of the pipeline. Thus the matrix generation is performed using low rank techniques while the arithmetic relies on the dense approach.

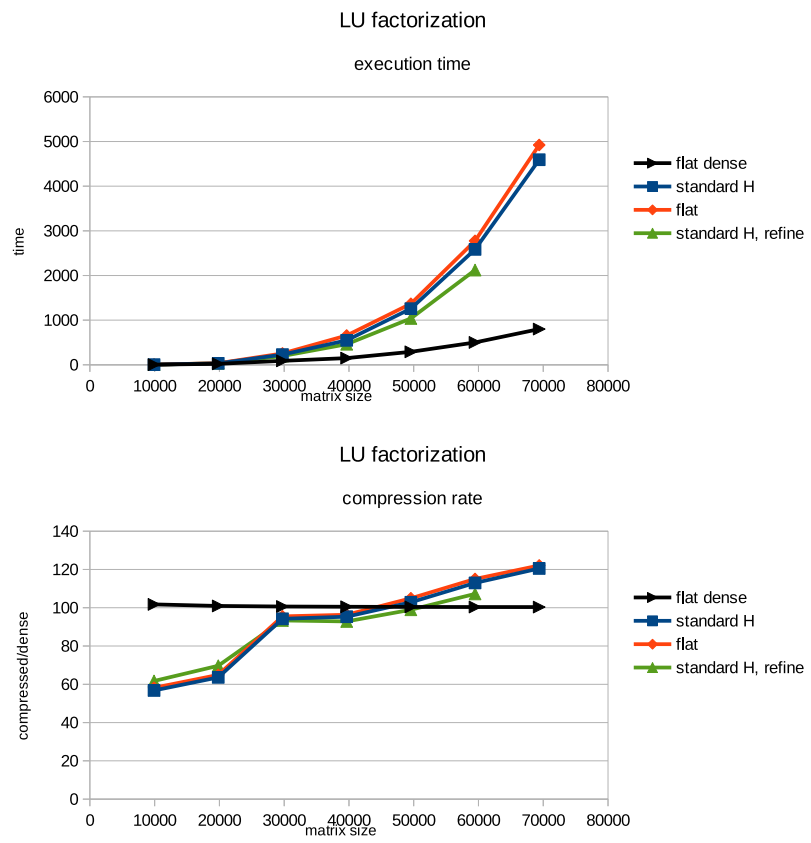

(a)

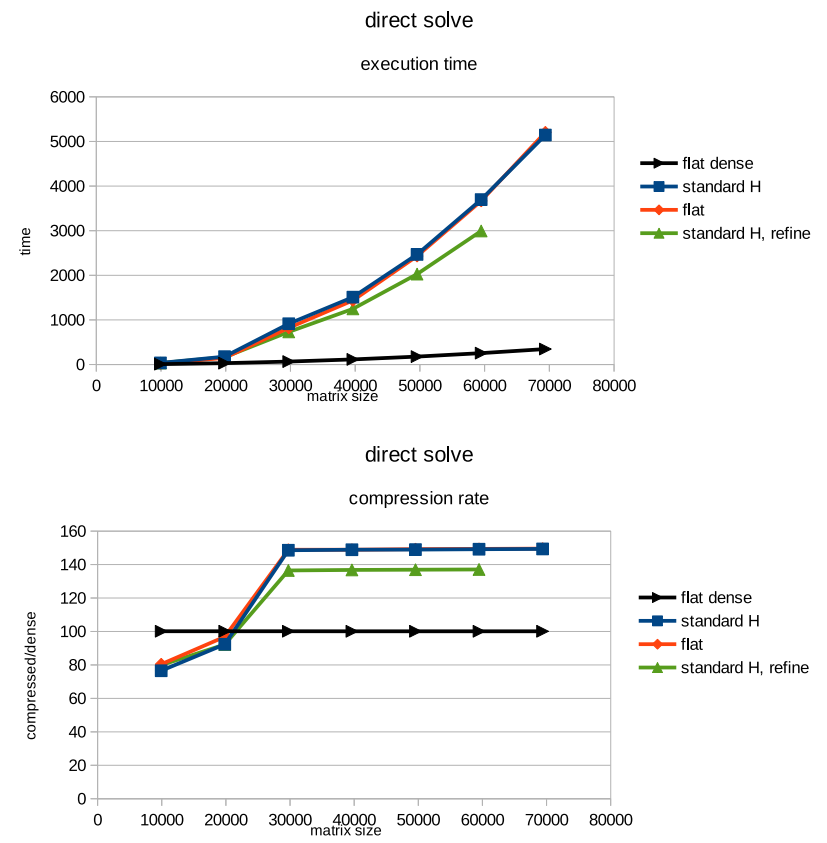

(b)

Figure 3: Execution time and compression rate of the $C_{m m}$ factorization (a) and direct solve (b) depending on the matrix size for different flavors of low rank approach. The increase of the matrix size is done by adding wavefront sensors (the number of sub-apertures along the diameter is constant). The wave-front noise is set as a constant.

Performed on Shihab.

\subsection{Matrix generation}

As mentioned in the 2.6, at ELT scale, the covariance matrix generation is likely to become a bottleneck for the application. We looked at low-rank format in order to improve this operation. At first, the WFS noise defined as a constant and as the software evolved, this noise was upgraded to take into account atmospheric and laser parameters. we present both cases hereafter. 


\subsubsection{Constant wavefront noise}

A first assessment of matrix generation was performed with a constant WFS noise, for the different low-rank formats for various matrix sizes, which is increased by adding wavefront sensors. The results, presented in the figure 4 shows similar performances for the different low-rank formats tested, in terms of computation time, allowing up to $30 \%$ of computation time reduction for the matrix generation, and compression rate, which is not affected by the number of wavefront sensors, remaining under $70 \%$. That last point indicates that the low-rank structure lies in the wavefront sensor blocks. We also noticed that the performance depends on the number of subapertures along the telescope diameter. In the following we use ELT scale telescopes with the appropriate number of subapertures.

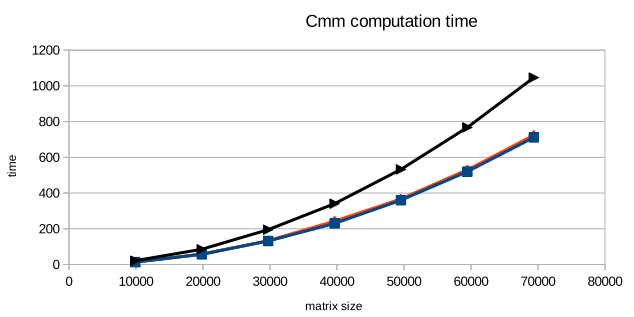

(a)

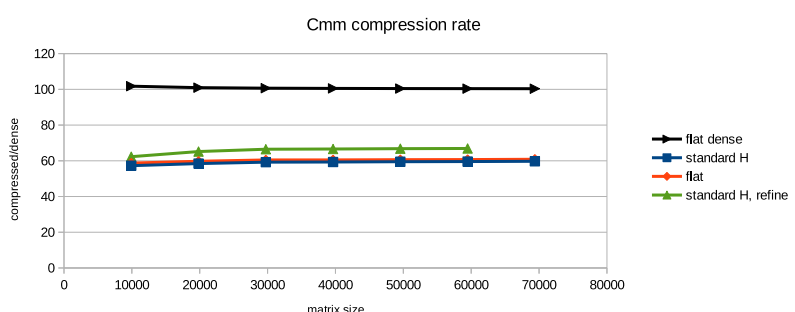

(c)

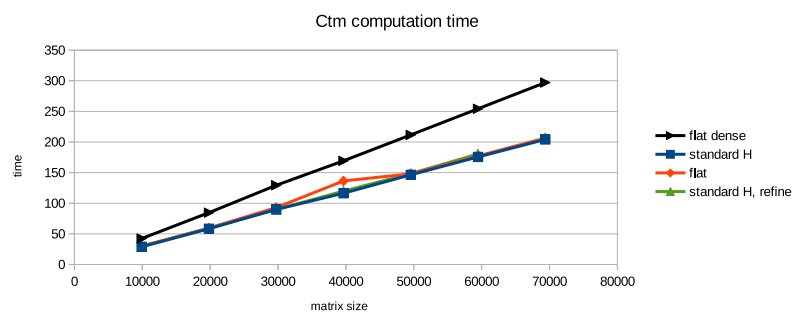

(b)

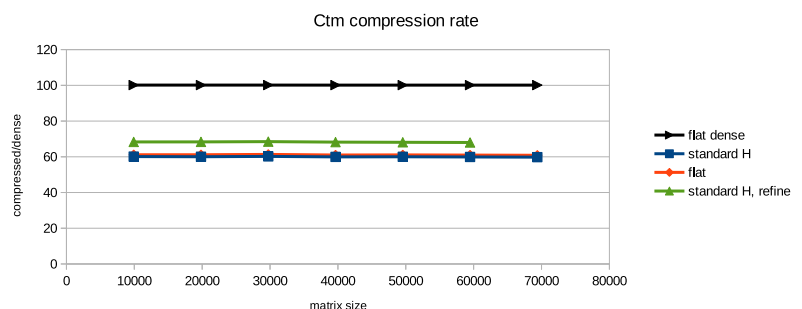

(d)

Figure 4: Execution time (on Shihab) of the Cmm generation (a) and Ctm (b) and compression rate for these matrices (respectively (c) and (d)) depending on the matrix size for different flavors of low-rank approach. The increase of the matrix size is done by adding wavefront sensors (the number of subapertures along the diameter is constant).

The wave-front noise is set as a constant.

As mentioned above, in the context of the low-rank approach, we refine the matrices into blocks. However, matrices can be partitioned in different ways ${ }^{7}$. Therefore, we introduced four of them, in an attempt to find the best one. The partitioning 0 is defined (Tab.1) with separated X and Y measurements that are still grouped by WFS. This is the partitioning used for the figure 4.

Table 1: Partitioning 0

\begin{tabular}{|c|cc|cc|c|cc|} 
WFS \# & \multicolumn{2}{|c|}{1} & \multicolumn{2}{|c|}{2} & \multicolumn{2}{|c|}{$\mathrm{N}$} \\
measurement type & $\mathrm{X}$ & $\mathrm{Y}$ & $\mathrm{X}$ & $\mathrm{Y}$ & $\ldots$ & $\mathrm{X}$ & $\mathrm{Y}$ \\
group indices & 1 & $\mathrm{~N}+1$ & 2 & $\mathrm{~N}+2$ & $\ldots$ & $\mathrm{N}$ & $2 \mathrm{~N}$
\end{tabular}

The partitioning 1 groups the $\mathrm{X}$ and Y measurements of a given WFS (Tab.2).

Table 2: Partitioning 1

\begin{tabular}{|c|cc|cc|c|cc|} 
WFS \# & \multicolumn{3}{|c|}{1} & \multicolumn{2}{|c|}{} & \multicolumn{3}{|c}{$\mathrm{N}$} \\
measurement type & $\mathrm{X}$ & $\mathrm{Y}$ & $\mathrm{X}$ & $\mathrm{Y}$ & $\ldots$ & $\mathrm{X}$ & $\mathrm{Y}$ \\
group indices & 1 & 1 & 2 & 2 & $\ldots$ & $\mathrm{N}$ & $\mathrm{N}$
\end{tabular} \mid

The partitioning 2 joins X measurements for all WFS on one side and all Y measurements for all WFS on the other (Tab.3). 
Table 3: Partitioning 2

\begin{tabular}{|c|cc|cc|c|cc|} 
WFS \# & \multicolumn{3}{|c|}{1} & \multicolumn{2}{|c|}{2} & \multicolumn{2}{|c|}{$\mathrm{N}$} \\
measurement type & $\mathrm{X}$ & $\mathrm{Y}$ & $\mathrm{X}$ & $\mathrm{Y}$ & $\ldots$ & $\mathrm{X}$ & $\mathrm{Y}$ \\
group indices & 1 & 2 & 1 & 2 & $\ldots$ & 1 & 2
\end{tabular}

The partitioning 3 groups all measurements of all WFS (Tab.4).

Table 4: Partitioning 3

\begin{tabular}{|c|cc|cc|c|cc|} 
WFS \# & \multicolumn{3}{|c|}{1} & \multicolumn{3}{|c|}{2} & \multicolumn{3}{|c}{$\mathrm{N}$} \\
measurement type & $\mathrm{X}$ & $\mathrm{Y}$ & $\mathrm{X}$ & $\mathrm{Y}$ & $\ldots$ & $\mathrm{X}$ & $\mathrm{Y}$ \\
group indices & 1 & 1 & 1 & 1 & $\ldots$ & 1 & 1
\end{tabular}

We report the performances of this low-rank approach for various generation accuracies and minimum block size, including the error of the approximated reconstructor, an estimation of the reconstructor performance in terms of Strehl ratio (compared to a full dense execution) and the matrix generation time.

A test on the error resulting from the low-rank approximation for a fixed matrix size (Fig. 5) show almost identical results for partitioning 0 and 1 for the error on both Cmm and ToR: the error increases regularly as generation accuracy decreases (to be expected), but in case of the ToR we notice a jump when the accuracy is lowered from $10^{-5}$ and $10^{-4}$. The partitionings 2 and 3 have a much larger Cmm error than the firsts two partitionings, and the ToR error in this case does not exhibit a clear trend. For this reason, we focused on the first two partitionings.
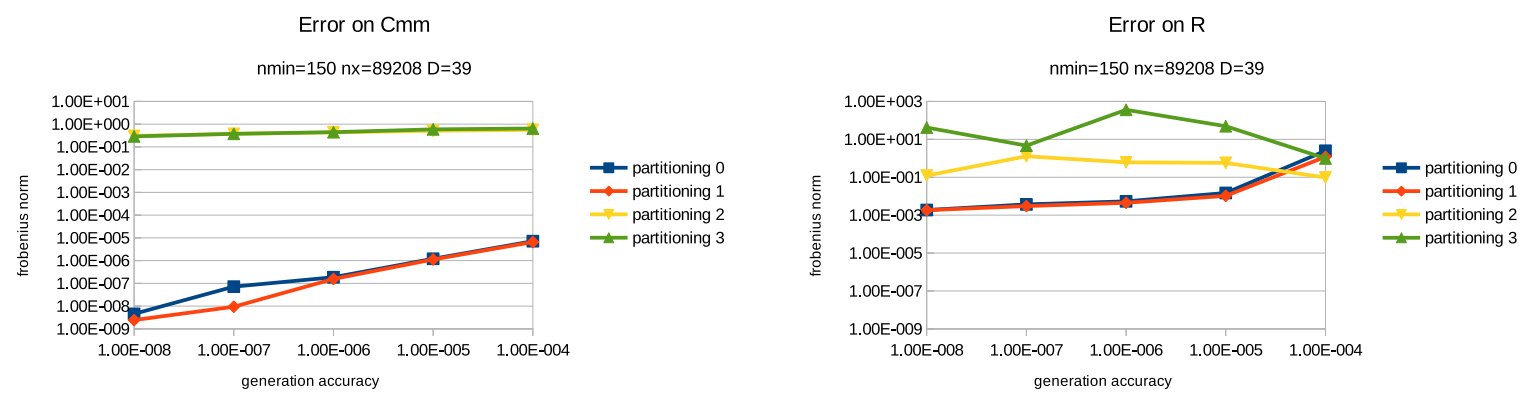

Figure 5: Error on the reconstructor and Cmm (Frobenius norm) computed with low-rank matrices construction and dense arithmetic compare to the full dense computation for a fixed matrix size and different accuracies. the wavefront noise is set as a constant.

Performed on Shihab.

Another experiment was performed using the end-to-end simulation tool COMPASS $\left[{ }^{10}\right]$ to estimate the performance of approximated tomographic reconstructors. We proceed in a few steps, the first one is to generate covariance matrices in either dense or low-rank (with various accuracies), then the corresponding reconstructor is computed using dense arithmetic and finally these reconstructors are inserted into the simulator. The different reconstructors are compared based on the Strehl ratio (on a long exposure of a thousand frames) provided by COMPASS.

We increased the matrix size by adding WFS, the objective being to keep a constant number of subapertures on the telescope diameter. Although it might not be the most relevant approach in terms of AO system dimensioning, we know the low-rank structure lies in the WFS blocks and increasing only the number of WFS will modify not this structure. Furthermore, it was not possible to go to the full ELT scale due to the too large memory footprint, so we limit the number of WFS to 5, and since the first WFSs are far away from the TS direction, the Strehl ratio stays low, but the point here was to assess the generation accuracy threshold and since the low-rank structure lies in the WFS blocks, the clear cut in accuracy we observe will also appear for a full ELT scale system with 

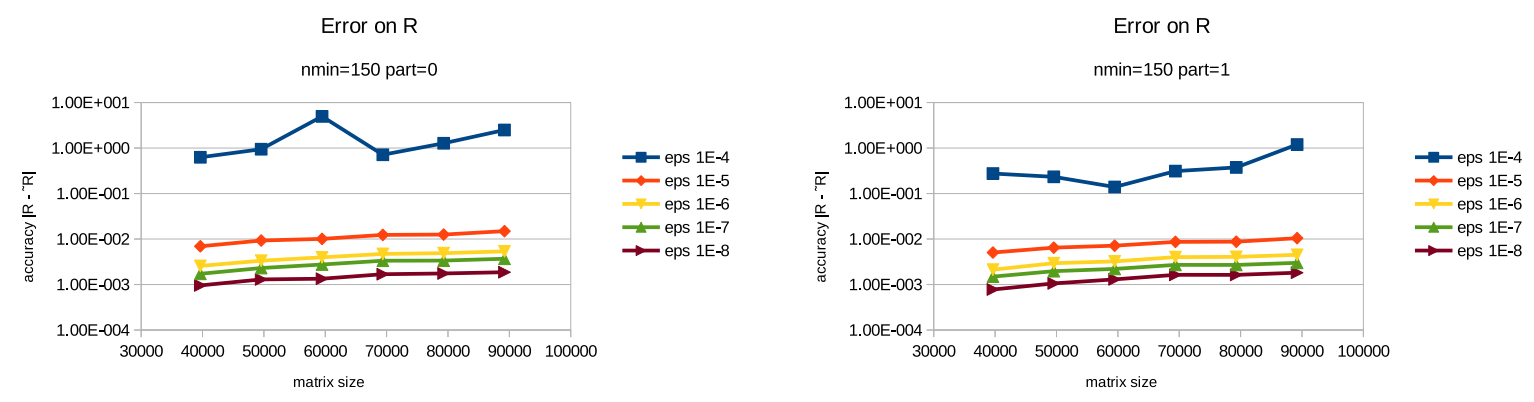

Figure 6: Reconstructor error (Frobenius norm) compared to dense computation for different accuracies, with an admissibility parameter of 2 . The reconstructor is computed from low-rank matrices construction and dense arithmetic.On a $39 m$ diameter telescope with 78 subapertures along the diameter. The wave-front noise is set as a constant.

Performed on Shihab.

10 WFS, whereas the Strehl ratio will increase as the WFS cover a larger area. In the results presented in Tab.5, even though as discussed above, the Strehl ratios are too low to be really significant, we observe a clear cut. Reconstructors computed from covariance matrices generated with an accuracy of $10^{-4}$ or better result in performances equivalent to the full dense approach, while an accuracy of $10^{-3}$ does not bring any correction to the optical system. Therefore, the accuracy threshold to generate the covariance matrices is between $10^{-4}$ and $10^{-3}$ for this experiment. Based on these figures and the graphs comparing the accuracy of the reconstructor for the different ordering (Fig. 6), the partitioning 0 should have a similar behavior, and even though the error on the reconstructor seems less predictable, an accuracy of $10^{-5}$ or $10^{-4}$ with the partitioning 2 .

Table 5: Estimation of the reconstructor performance with the end-to-end simulation tool COMPASS. Simulation of an LTAO system on a $38 \mathrm{~m}$ diameter telescope. The reconstructor are computed with the partitioning 1

\begin{tabular}{|c|c|c|c|c|c|c|}
\hline \multirow{2}{*}{ Number of WFS } & \multirow{2}{*}{ Number of measurements } & \multicolumn{4}{|c|}{ Strehl ratio(long exposure:1000 iterations) } \\
\cline { 3 - 6 } & & \multirow{2}{*}{ dense } & \multicolumn{4}{|c|}{ low-rank, generation accuracy: } \\
\cline { 3 - 6 } & 26208 & $6 \%$ & $6 \%$ & $5 \%$ & $5 \%$ & $5 \%$ \\
\hline 3 & 34944 & $7 \%$ & $8 \%$ & $7 \%$ & $7 \%$ & $0 \%$ \\
\hline 4 & 43680 & $8 \%$ & $8 \%$ & $7 \%$ & $7 \%$ & $0 \%$ \\
\hline 5 & & &
\end{tabular}

\subsubsection{More realistic wavefront noise}

As we developed the software, the model function to generate covariance matrices was changed to include a more realistic wavefront noise ${ }^{11}$ than the model using a constant presented above. This modification takes into account the throughput of the atmosphere, the laser flux and the elongation of the lasers' spots. As a consequence of this modification, the partitioning is now limited to the predefined blocking which only allows a few subblocks to be generated by low-rank methods and degrade the ability of low-rank techniques to speed up the matrix generation (compared to the dense generation).

We also notice the impact of the parameter used to simulated the fact that LGS cannot measure the tip-tilt and focus on the compression. this is done using a constant added on relevant blocks of the covariance matrix. A realistic value of this constant would be in the range of 0.1 to 10 . We choose this interval because this constant must be significantly greater than the diagonal values of the covariance matrix to actually invalidate the tip-tilt information, but not too large. The reason is that the numerical precision is limited and a too large value of this constant will overwhelm the actual covariance value. The impact mentioned above is the following, as this constant increase, the covariance matrix condition number grows quickly. Hence theses values result in a high condition number hindering compression. Since this constant is only applied to the LGS, the compression depends on the number of LGS : an increasing number of LGS leads to much less compression (this can be highlighted by replacing some of the LGS by NGS). 
We also notice a few variations of the behavior when the this constant grows up (from 0.01 to 0.1 and 1 ). In the case of the partitioning 2 and 3, the computation time decreases, the compression increases but the error on the reconstructor increases. The partitioning 0 and 1 remain about the same.

With this new wavefront noise and this constant value, it became more difficult to outperform the dense approach, shown in the following figure (Fig. 7). This benchmark present the matrix generation time depending on the matrix size. The partitioning 1 is faster than partitioning 0 for the $C_{m m}$ or $C_{t m}$ generation but is still slower than the dense approach.
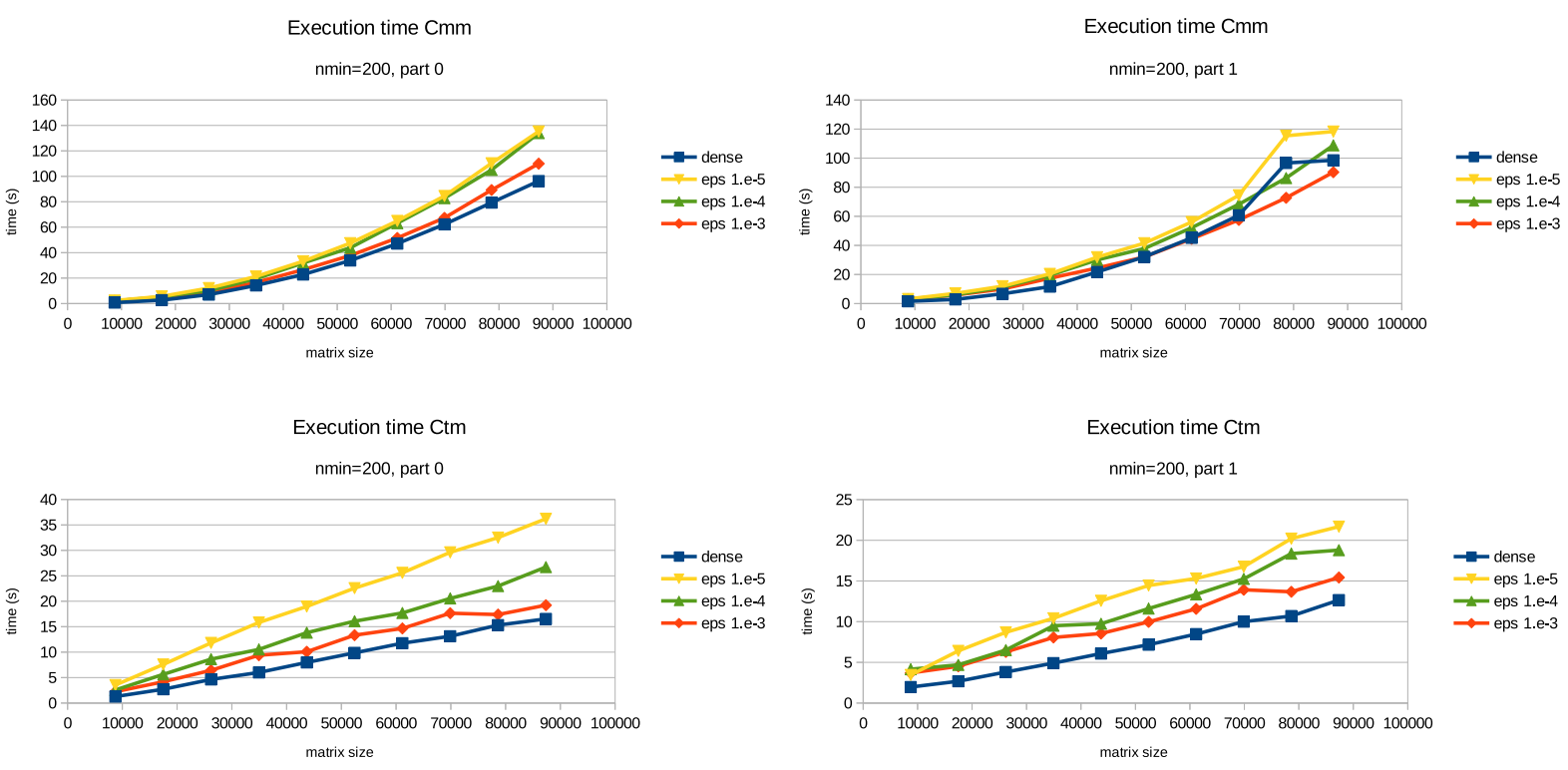

Figure 7: Execution time (on Flamingo) to generate the covariance matrices $\left(C_{m m}\right)$ according to the matrix size for accuracies and partitioning. Eta $=2$ nmin $=200$.

In order to understand more, we proceed to compute the covariances matrices of each layer independently. In the following graphs (Fig. 8), we compute for various altitude the covariance matrices with a fixed outer scale (L0) and strength (cn2). The execution time is slightly decreasing with the altitude for the partitioning 0 and 1 while it is increasing for the partitioning 2 and 3 . This is particularly visible in the case of the $C_{m m}$ where for the ground layer, the partitioning 2 and 3 are faster than the 0 and 1 but takes at least twice as much time for the higher layers. The error on the $C_{t m}$ is increasing with the altitude for all the partitioning with similar values even though the partitioning 0 and 1 have a better estimation. The error on the $C_{m m}$ is constant for the partitioning 0 and 1 and decreasing for the partitioning 2 and 3 but the error the these two last is far greater. The altitude does not seems to have a significant influence on the behavior of the matrix generation for the partitioning 0 and 1 in this case.

In the Figure (9), we try various combination of outer scale and altitude. The slight improvement depending on the altitude previously mentioned now has several trends depending on the value of the outer scale. Furthermore, in all cases, the higher the outer scale, the better the performance: an outer scale of $25 \mathrm{~m}$ (which is the default value used for all the previous results)result in slower execution than the dense format while an outer scale of $100 \mathrm{~m}$ reduce the computation time by half.

Since the performance depends on the value of the outer scale, we used outer scale values extrapolate from an actual measurement campaign. ${ }^{12}$ The results (Fig. 10) show a dependence between the value of the outer scale and the performance of the low-rank approach. The low-rank matrix generation outperforms the dense approach when the outer scale value is greater than $40 \mathrm{~m}$ or smaller than $20 \mathrm{~m}$, this is particularly visible for the second profile. We also notice that this interval is varying slightly with the altitude. A possible explanation would be that the low-rank approach has better performance when the outer scale is not of the same order as the distance 

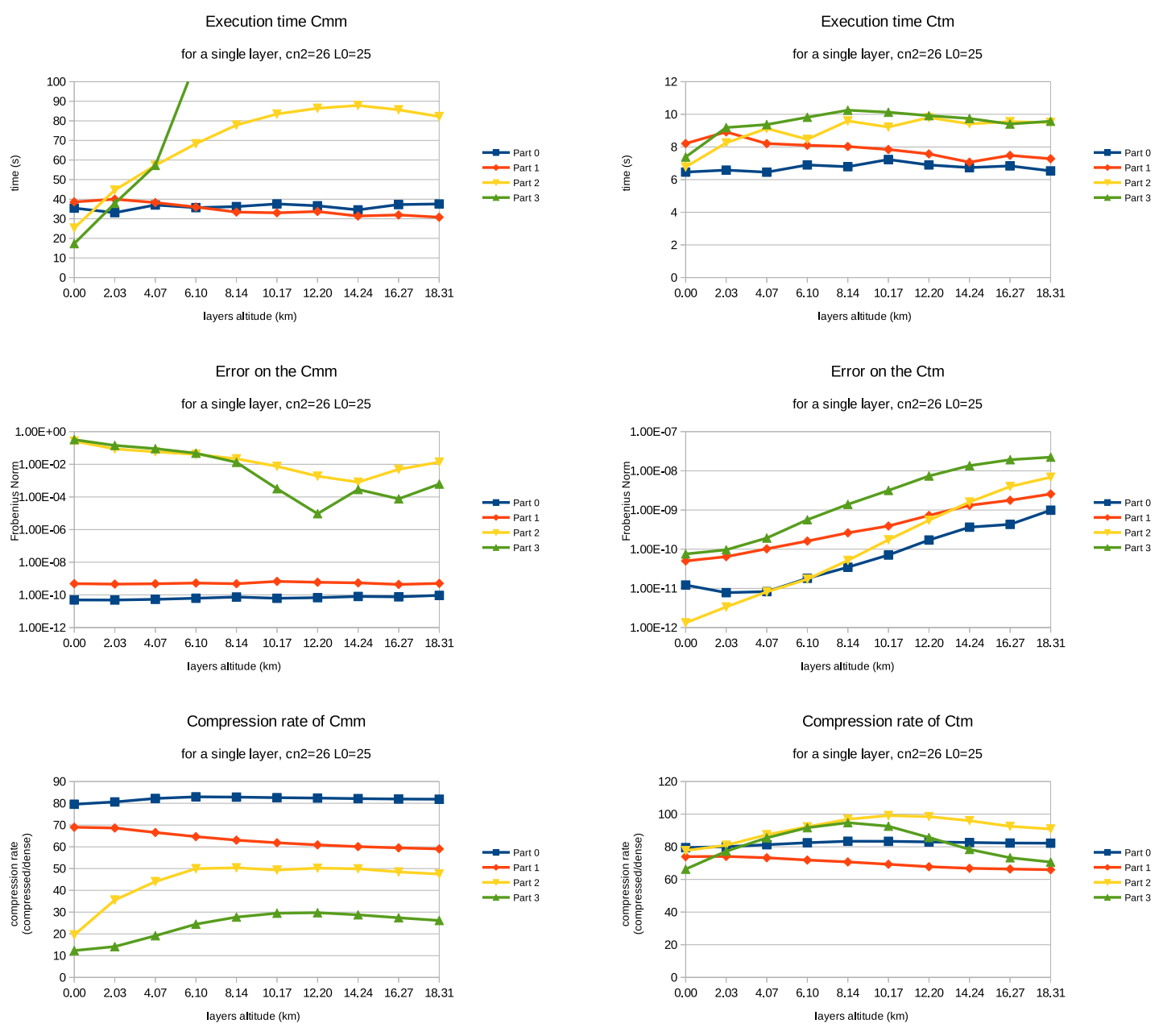

Figure 8: Computation time, error and compression rate of the $C_{m m}$ (left) and $C_{t m}$ (right) for a single layer according to this layer altitude. On a $38 \mathrm{~m}$ diameter telescope with 9 WFSs and 76 subapertures along the diameter. Eps $=10^{-4}$, eta $=0.5$, nmin $=200$, lgs_cst $=0.01$. The "ref" curves correspond to the computation time using dense format. (performed on Niravna).

between the subapertures. The $C_{t m}$ has a similar behavior as the $C_{m m}$ but the improvement of the low-rank compare to the dense is not as good as the $C_{m m}$ case. One can show that the matrix generation runtime using

Table 6: $C_{m m}$ computation time for atmospheric parameter extrapolate from an actual measurement campaign ${ }^{12}$

\begin{tabular}{|c|c|c|c|c|c|c|}
\hline & \multicolumn{3}{|c|}{$C_{m m}$} & \multicolumn{3}{c|}{ Ctm } \\
\cline { 2 - 7 } & dense & partitioning 0 & partitioning 1 & dense & partitioning 0 & partitioning 1 \\
\hline maximum L0, 07/12/21 & 361.29 & 216.63 & 182.61 & 67.6 & 37.01 & 35.12 \\
\hline average L0, 07/12/21 & 373.49 & 390.43 & 340.30 & 72.1 & 76.12 & 69.48 \\
\hline
\end{tabular}

this low-rank approach depends on the strength of the strongest layers, that is if the outer scale of theses layers belongs to the unfavourable interval then the low-rank and dense approaches have similar performances, if on the contrary, the outer scales have favourable values then significant improvement are observed. In the case of the profile 1 in the Table (6) the low-rank approach halve the runtime compared to the dense format. 

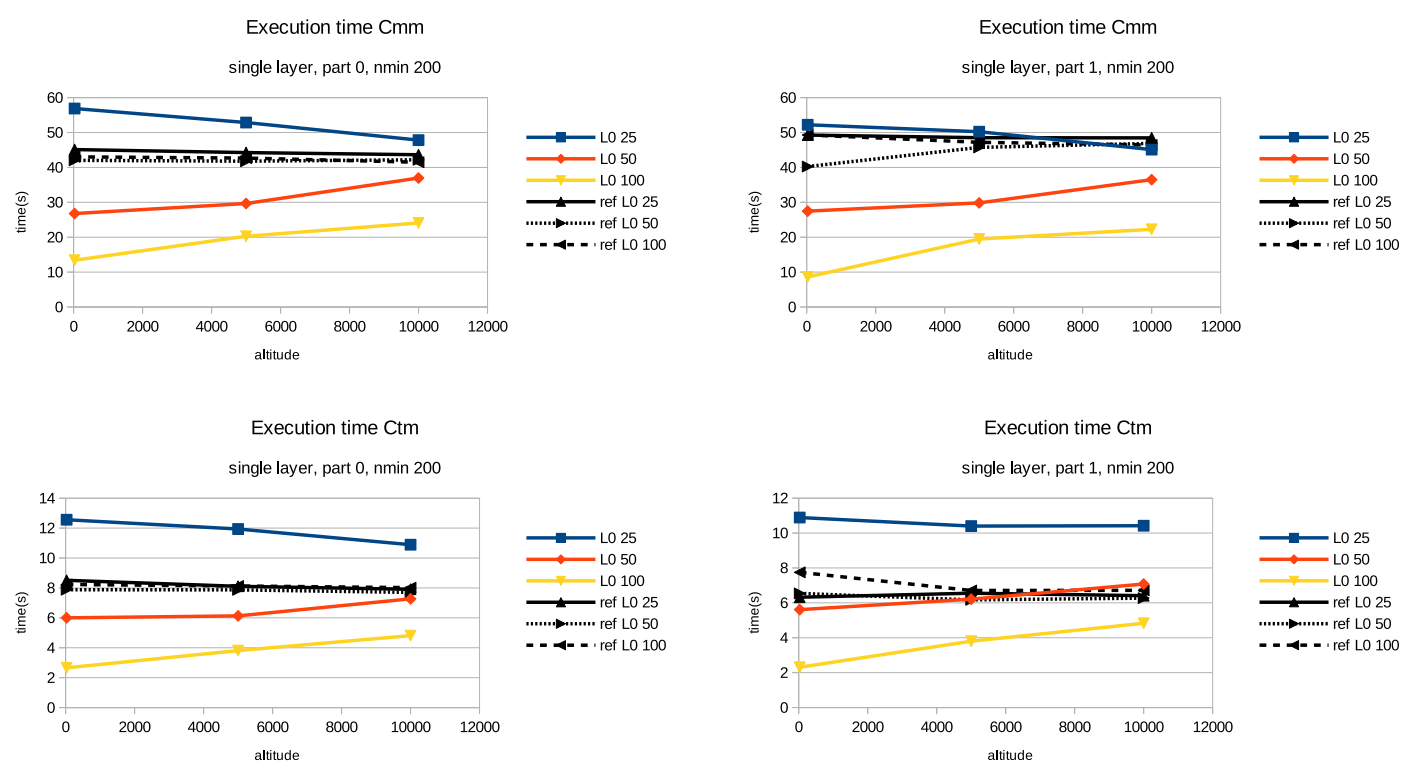

Figure 9: Execution time (Flamingo)on to generate the covariance matrices $\left(C_{m m}\right)$ according to the matrix size for accuracies and partitioning. eta $=2 \mathrm{nmin}=200$. 
LO profile

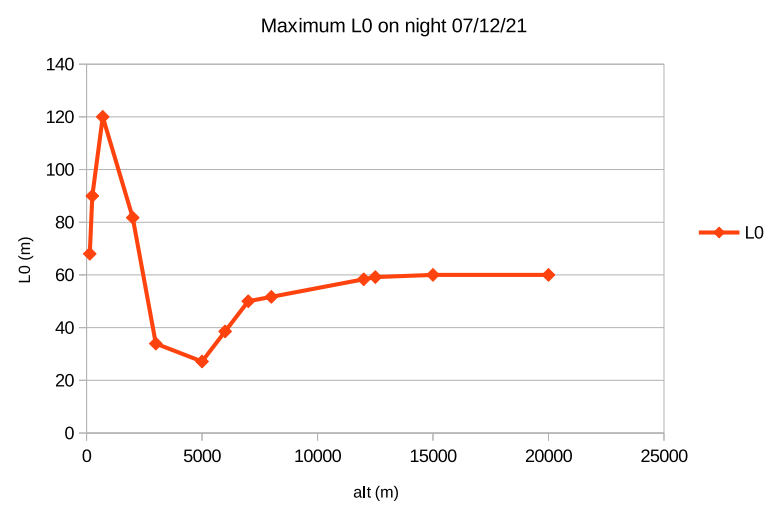

LO profile

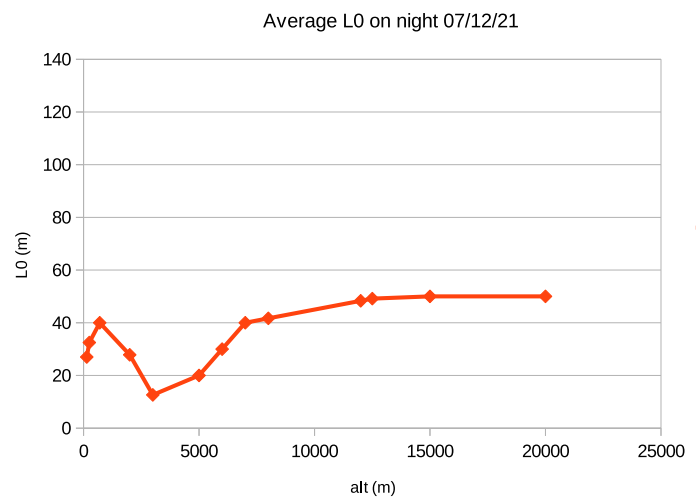

Computation time

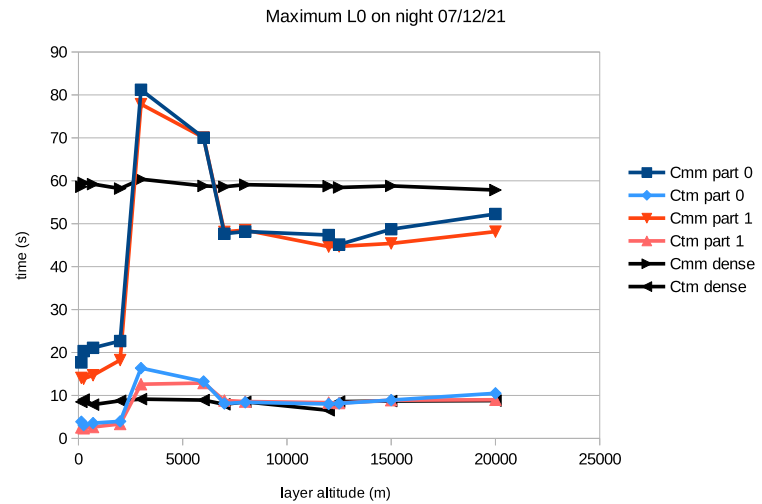

Computation time

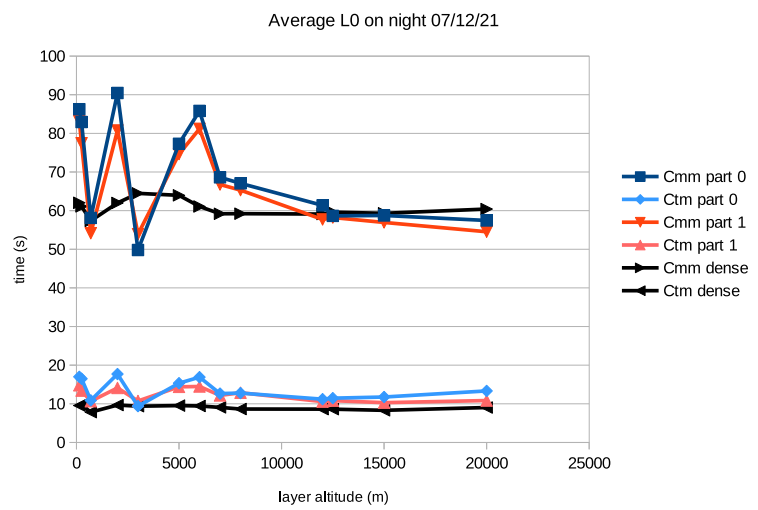

Figure 10: On the left side is the outer scale according the layer altitude, on the right the computation time of a single layer according to its altitude. Using a 38m telescope with 10 WFSs and 76 subapertures (along the diameter). The following benchmark introduces a variable L0, extrapolated by linear interpolation from an actual measurement campaign ${ }^{12}$, using the average values on night $07 / 12 / 21$ and the maximum L0 on night $07 / 12 / 21$ the cn2 profile is identical for the three figures above. 


\section{CONCLUSION}

We describe in this paper a supervision strategy for different kind of tomographic AO, aiming for the ELT scale, and exploring low-rank approach, focusing on the Apply step of the pipeline as well as the matrix generation. The results presented here shows that if the low-rank approach does not bring improvement to the Apply step, the matrix generation, which at ELT scale is likely to be the dominant part, can largely benefit from it and achieve runtime reductions up to a factor of two relative to dense format. The low-rank approach could be can be considered for operation that does not belong to the supervisor module. That is the case of the Real Time Controller responsible for adapting the Deformable Miror (DM) shape in order to compensate for the effect of the turbulence. It involves a matrix-vector multiply that transforms the actual measurements from the telescope WFS into command for the DM. The matrix under consideration is the tomographic reconstructor, output of the supervisor module. By speeding up the matrix-vector multiply, the $\mathcal{H}$-matrix linear algebra is considered promising for real-time adaptive optics.

\begin{tabular}{l|c|l}
\hline System name & Operating system & hardware \\
\hline Flamingo & Ubuntu 16.04.2 LTS & $\begin{array}{l}\text { Two sockets 28 cores Intel(R) Xeon(R) Skylake } \\
\text { Platinum 8176 CPU @ 2.10GHz }\end{array}$ \\
\hline Nirvana & Ubuntu 16.04.3 LTS & $\begin{array}{l}\text { Two sockets 20 cores Intel(R) Xeon(R) Broadwell } \\
\text { E5-2698 v4 @ 2.20GHz }\end{array}$ \\
\hline shihab & Ubuntu 14.04.5 LTS & $\begin{array}{l}\text { Two sockets 18 cores Intel(R) Xeon(R) Haswell } \\
\text { E5-2699 v3 @ 2.30GHz }\end{array}$ \\
\hline
\end{tabular}

Table 7: Computing systems characteristics

\section{REFERENCES}

[1] Vidal, F., Calibrations et reconstruction tomographique en optique adaptative multi-objet pour l'astronomie : Application au démonstrateur CANARY, PhD thesis, Observatoire de Paris (2009.).

[2] Gendron, E., Charara, A., Abdelfattah, A., Gratadour, D., Keyes, D., Ltaief, H., Morel, C., Vidal, F., Sevin, A., and Rousset, G., "A novel fast and accurate pseudo-analytical simulation approach for MOAO," (2014).

[3] Morel, C., Gendron, E., Gratadour, D., Sevin, A., and Rousset, G., "Pseudo-analytic simulation of woofertweeter MOAO system: application to MOSAIC," (2016).

[4] Ltaief, H., Charara, A., Gratadour, D., Doucet, N., Hadri, B., Gendron, E., and Keyes, D., "Real-Time Massively Distributed Multi-Object Adaptive Optics Simulations for the European Extremely Large Telescope,"

[5] "HLIBpro." http://www.hlibpro.com/. (Accessed: 1 March 2018).

[6] Hackbusch, W., "A Sparse Matrix Arithmetic Based on H -Matrices. Part I: Introduction to H -Matrices," Computing 62, 89-108 (1999).

[7] Hackbusch, W., [Hierarchical matrices : algorithms and analysis], vol. 49 of Springer series in computational mathematics, Springer, Berlin (2015).

[8] Weisbecker, C., Improving multifrontal solvers by means of algebraic Block Low-Rank representations, $\mathrm{PhD}$ thesis, Institut National Polytechnique de Toulouse (2013).

[9] Bebendorf, M., "Approximation of boundary element matrices," Numerische Mathematik 86, 565-589 (Oct 2000).

[10] Ferreira, F., Gratadour, D., Sevin, A., Doucet, N., Gendron, E., Vidal, F., Deo, V., and Durand, S., "Realtime end-to-end AO simulations at ELT scale on multiple GPUs with the COMPASS platform," (2018).

[11] Robert, C., Conan, J., Gratadour, D., Schreiber, L., and Fusco, T., "Tomographic wavefront error using multi-LGS constellation sensed with Shack-Hartmann wavefront sensors," J. opt Soc. Am A27, A201-A215 (2010). 
[12] Ali, W. D., Ziad, A., Berdja, A., Maire, J., Borgnino, J., Sarazin, M., Lombardi, G., Navarrete, J., Ramio, H. V., Reyes, M., Delgado, J. M., Fuensalida, J. J., Tokovinin, A., and Bustos, E., "Multi-instrument measurement campaign at Paranal in 2007 Characterization of the outer scale and the seeing of the surface layer," Astronomy Astrophysics (2010). 